\title{
Extracorporeal Shockwave Lithotripsy versus Ureteroscopy for Distal Ureteric Calculi: Efficacy and Patient Satisfaction
}

\author{
Ibrahim F. Ghalayini, Mohammed A. Al-Ghazo, Yousef S. Khader \\ School of Medicine, Jordan University of Science \& Technology, King Abdullah University \\ Hospital, Irbid, Jordan
}

\begin{abstract}
Objective: We compared the efficacy of extracorporeal shock wave lithotripsy (ESWL) and ureteroscopy (URS) for the treatment of distal ureteral calculi with respect to patient satisfaction.

Materials and Methods: This is a prospective study where a total of 212 patients with solitary, radiopaque distal ureteral calculi were treated with ESWL $(\mathrm{n}=92)$ using Dornier lithotriptor S (MedTech Europe GmbH) or URS $(\mathrm{n}=120)$. Patient and stone characteristics, treatment parameters, clinical outcomes, and patient satisfaction were assessed for each group. Results: The 2 groups were comparable in regard to patient age, sex, stone size, and side of treatment. The stone-free status for ESWL and URS at 3 months was $81.5 \%$ and $97.5 \%$, respectively ( $\mathrm{p}<0.0001$ ). In addition, $88 \%$ of patients who underwent ESWL versus $20 \%$ who underwent URS were discharged home the day of procedure. Minor complications occurred in $3.3 \%$ and $8.3 \%$ of the ESWL and URS groups, respectively $(\mathrm{p}=0.127)$. No ureteral perforation or stricture occurred in the URS group. Postoperative flank pain and dysuria were more severe in the URS than ESWL group, although the differences were not statistically significant $(\mathrm{p}=0.16)$. Patient satisfaction was high for both groups, including $94 \%$ for URS and $80 \%$ for ESWL ( $p=0.002)$.

Conclusions: URS is more effective than ESWL for the treatment of distal ureteral calculi. ESWL was more often performed on an outpatient basis, and showed a trend towards less flank pain and dysuria, fewer complications and quicker convalescence. Patient satisfaction was significantly higher for URS according to the questionnaire used in this study.
\end{abstract}

Key words: ureteral calculi; ureteroscopy; extracorporeal shockwave lithotripsy

Int Braz J Urol. 2006; 32: 656-67

\section{INTRODUCTION}

Urinary lithiasis can cause a greater or lesser degree of obstruction of the lower ureter, depending on the size of the calculus, urothelial edema and the degree of impaction, requiring instrumental treatment, sometimes as an urgent procedure. In the past 25 years, the treatment of these calculi has evolved from ureterolithotomy to ureterorenoscopy URS (1), extracorporeal shockwave lithotripsy (ESWL) (2), and endoscopic lithotripsy $(3,4)$.
Advances in the design of the ureteroscope and ongoing development in ESWL have greatly impacted the management of ureteric stones (5). The indications for ureteroscopic lithotripsy have increased with smaller semi-rigid ureteroscopes and reliable laser technology and the production of more robust flexible instruments has further expanded the indications for endoscopic intervention. Despite the definite success of endourological stone treatment, ongoing questions about optimum management remain debated among urologists. 
ESWL and URS are currently accepted treatment modalities for distal ureteral calculi. Some authors $(6,7)$ favor ESWL while others (8-10) prefer URS. For both treatment modalities stone-free rates of more than $90 \%$ have been reported $(7,9,10)$.

The American Urological Association Ureteral Stones Clinical Guidelines Panel has found both to be acceptable treatment options for patients, based on the stone-free results, morbidity, and retreatment rates for each respective therapy. However, costs and patient satisfaction or preference were not addressed (11).

We aim to compare herein the efficacy and safety of ESWL and URS for distal ureteral calculi with respect to patient satisfaction.

\section{MATERIALS AND METHODS}

A total of 212 patients undergoing therapy for distal ureteral calculi between January 2001 and December 2004 were entered into the study. Patients presented with radiodense ureteral stones distal to the bony pelvis on excretory urogram or computed tomography (CT) which had not passed spontaneously within 3 weeks. Patients were included in the study only if the intervention, either ESWL or URS, was the primary modality and there was a minimum follow-up period of 3 months. Patients for whom either therapeutic modality was contraindicated because of pregnancy, urinary tract infection, coagulation disorders or previous ureteral reimplantation were excluded from the study.

After defining the indications of treatment, the patients were made aware of both the modalities of treatment and their probable complications. The need for anesthesia, stent, urethral manipulation, possible complications, need for repeated follow up especially after ESWL, and the cost factor involved, were explained to the patient. The patients were then asked to choose the mode of treatment.

ESWL was performed using the Dornier lithotriptor S (MedTech Europe $\mathrm{GmbH}$ ). All patients were positioned prone and the calculi were localized with fluoroscopic guidance. All patients were given sedatives and analgesics and the level of shockwave energy was progressively stepped up till satisfactory stone fragmentation within the patient's comfort. URS was performed with rigid $8 \mathrm{~F}$ Wolf ureteroscope following dilatation of ureteric orifice if needed. The stones were either extracted via basket or forceps, or disintegrated with the Pneumatic lithotripsy lithotriptor. Placement of a ureteral stent at the conclusion of the procedure was left to the discretion of the treating surgeon. Upon completion of the procedure, fluoroscopic imaging was performed to determine whether the ureter was stone-free. All patients were administered prophylactic antibiotic.

Complete stone clearance was assessed at three months follow up. Patients were followed-up to assess the success rates and complications of the two procedures. The follow up schedule was similar in both groups of patients. Plain X-rays were obtained 1, 2, 4 and 6 weeks after discharge and thereafter if residual fragments persisted. Obstruction of the upper urinary tract was excluded from diagnosis with the help of ultrasonography. In case of recurrent ureteral colic or if calculi failed to pass within 6 weeks ureteroscopic stone removal was performed. Treatment failure was based on the need for further surgical intervention during follow-up or failure to become stone-free within 3 months (7). At initial follow-up, patients were given a questionnaire, which we use for all patients with urolithiasis in our center based on published data about the factors that influenced patient satisfaction $(7,9)$ (Table-1). Those with total score of 8 or less were considered satisfied with the procedure. The efficiency quotient (EQ) was calculated using the formula: Stone free $(\%) \times 100 /$ $(100+$ retreatment rate $(\%)+$ rate of auxiliary procedures (\%)) (12).

Data were analyzed using Statistical Package for Social Sciences (SPSS, version 11.5). Pearson's chi-square, student t-test, Mann-Whitney U test was used where appropriate and $p<0.05$ was considered statistically significant.

\section{RESULTS}

One hundred and twenty patients were treated with URS (male/female: 85/35), while 92 (male/ 
Table 1 - Postoperative questionnaire.

\begin{tabular}{lcccc}
\hline & $\mathbf{0}$ & $\mathbf{1}$ & $\mathbf{2}$ & $\mathbf{3}$ \\
\hline Postoperative symptoms: & & & & Moderate \\
$\quad$ Dysuria & No & Mild & Severe \\
Hematuria & No & Microscopic & Macroscopic -no clots & With clots \\
$\quad$ Loin pain & No & Mild & Moderate & Severe \\
Time to normal activity & $0-1$ day & $2-3$ days & $3-6$ days & $>6$ days \\
Global satisfaction & Yes & Not sure & No & No \\
Willingness to repeat & Yes & Not sure & No \\
Recommending the procedure & Yes & Not sure & &
\end{tabular}

female: 70/22) patients were treated by ESWL. Patient's age varied between 11 and 75 years, with maximum number of patients between 35 to 45 years of age. There were no significant differences in the mean age, sex ratio and stone size in both groups (Table-2). For the extracorporeal modality, i.e. ESWL, the mean stone size was $10.4 \pm 5.3 \mathrm{~mm}$ (range 4 to 27) (Table-2). In this group, $90 \%$ received intravenous sedation and $10 \%$ general anesthesia. Majority of the patients $(88 \%)$ had treatments as an outpatient procedure but all patients needed frequent follow-up visits. Only 4 patients $(4.3 \%)$ required pre-ESWL double pigtail stents for persistent ureteric colic not responding to conservative treatment. A total of 92 patients required 115 sessions of lithotripsy with average number of 3720 shock waves at $10-20 \mathrm{kV}$. Stone-free status at 1 month and 3 month were $67 \%$ $(n=62)$ and $81.5 \%(n=75)$, respectively (Figure-1). There were no major complications, although three patients (3.3\%) developed fever and infection. In total,
23 patients $(25 \%)$ required more than one session of ESWL for disintegration, whereas 17 patients (18.5\%) where ESWL failed were treated by URS for 16 cases and open ureterolithotomy for one patient with a hard $27 \mathrm{~mm}$ stone (Table-3). Of these, there were 4 cases of "steinstrasse" (4\%) after ESWL and only 2 were treated conservatively; the other 2 required URS. EQ at 3 months was 57 . Considerable differences with regard to patient satisfaction were noted with a mean score of $5.03 \pm 3.08$. Of the patients $74(80 \%)$ were satisfied and will recommend the procedure to the others while 18 (20\%) who required re-treatment or URS would opt for URS for recurrence (Table-3).

For the intracorporeal modality, i.e. URS with pneumatic lithotripsy, the mean stone size was $9.2 \pm$ $5.4 \mathrm{~mm}$ (range, 4 to 27) (Table-2). In this group, $60 \%$ of patients had general anesthesia, $25 \%$ local anesthesia and $15 \%$ intravenous sedation. The majority of the patients had treatments as an inpatient procedure $(80 \%)$ mainly for 'social' reasons, like difficulty

Table 2 - Baseline comparability of the 2 treatment groups.

\begin{tabular}{lccc}
\hline & ESWL & Ureteroscopy & p Value (t-test) \\
\hline N patients & 92 & 120 & $0.104^{*}$ \\
Mean age \pm SD (year) & $42.3 \pm 12.0$ & $45.3 \pm 14.0$ & $0.394 \dagger$ \\
Male to female ratio & $1: 0.3$ & $1: 0.4$ & $0.121^{*}$ \\
Mean stone size \pm SD (mm) & $10.4 \pm 5.3$ & $9.2 \pm 5.4$ & $0.979 \dagger$ \\
N stones Rt./Lt. side & $42 / 50$ & $55 / 65$ & \\
\hline
\end{tabular}

*t-test, $\dagger$ chi-square test. 


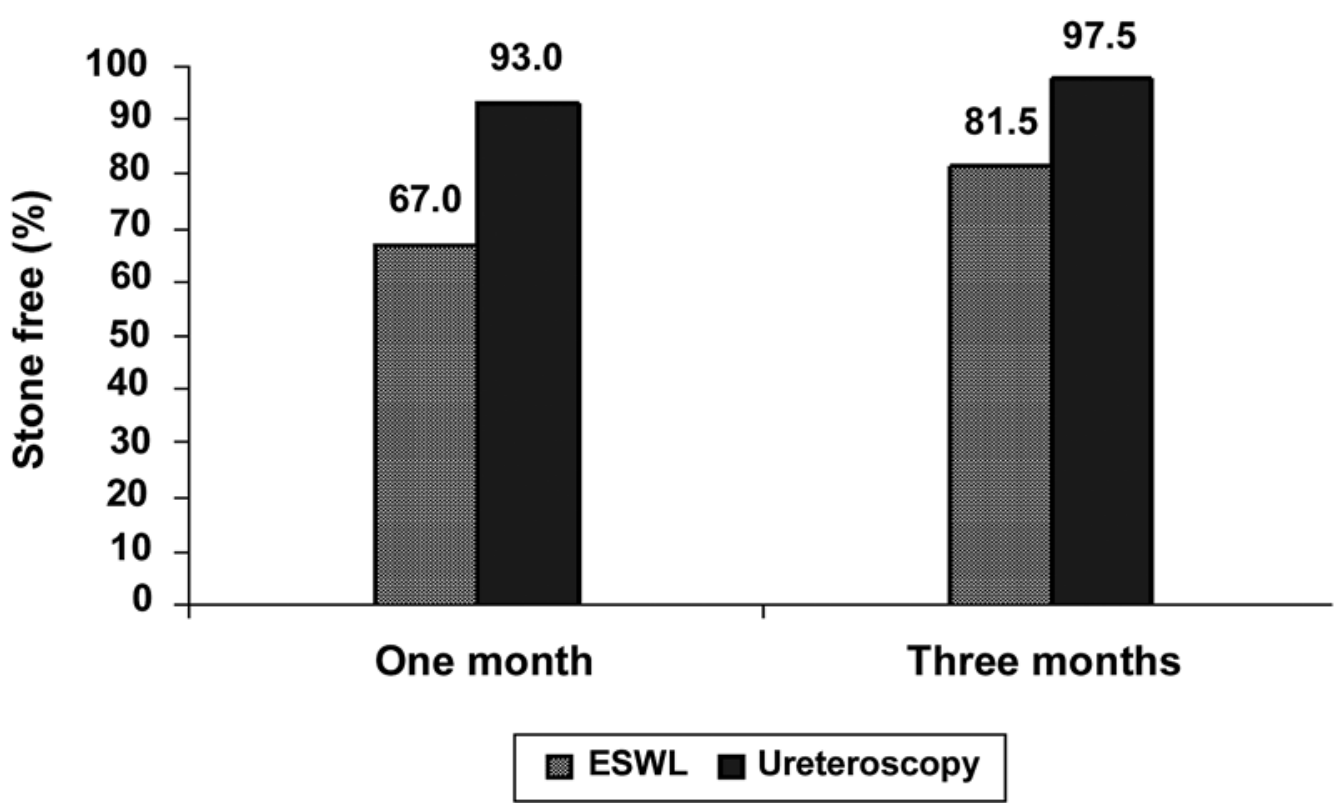

Figure 1 - Stone free rate at 1 month and 3 months.

in transport. Some of these were admitted for pain control, infection and stent-related symptoms but all patients needed less frequent visits for follow-up than ESWL. After URS, ureteric catheter or double J stent was kept in 41 patients $(34.2 \%)$ for 24 hours to 3 weeks. Of these, 12 patients (10\%) required postoperative double-J ureteric stenting due to high stone load. In 28 cases ( $23 \%$ ), the calculi could be extracted without fragmentation (forceps retrieval in 17 and basket retrieval in 11) and all other stones were fragmented using the Pneumatic lithotripsy lithotriptor. Repeat URS was however required in 8 patients (6.7\%) after 4 weeks (Table-3). EQ at 3 months was 89. In these patients the initial attempt of URS failed due to failure to adequately dilate the ureteric orifice in six and submucosal dissection with false passage

Table 3 - Results of ESWL versus Ureteroscopy.

\begin{tabular}{lccc}
\hline & ESWL & Ureteroscopy & p Value (t-test) \\
\hline N of auxiliary procedures & $17(18.5 \%)$ & $3(2.5 \%)$ & $<0.0001^{*}$ \\
& URS and open & ESWL and open & \\
Complications & $3(3.3 \%)$ & $10(8.3 \%)$ & $0.127^{*}$ \\
Mean follow-up \pm SD (weeks) & $5.8 \pm 3.0$ & $4.2 \pm 3.4$ & $<0.0001 \dagger$ \\
N of re-treatment & $23(25.0 \%)$ & $8(6.7 \%)$ & $<0.0001^{*}$ \\
Patient satisfaction & $74(80.4 \%)$ & $113(94.2 \%)$ & $0.002^{*}$ \\
Postoperative analgesia & $68(73.9 \%)$ & $104(86.7 \%)$ & $0.019^{*}$ \\
Mean period of analgesia \pm SD (days) & $1.9 \pm 1.5$ & $2.4 \pm 1.5$ & $0.029 \dagger$ \\
Efficiency quotient at 3 months & 57 & 89 & \\
\hline
\end{tabular}

* Chi-square test, $\dagger$ Mann-Whitney U test. 
in two patients. Open surgery was required for one of these patients who had a hard $25 \mathrm{~mm}$ stone. The proximal migration of calculus occurred in 2 patients (1.7\%) who were treated by ESWL. Mean hospital stay in URS was two days. With respect to complications, there were 6 cases $(5 \%)$ of infection in addition to 2 cases of proximal stone migration and 2 cases of submucosal dissection. No long-term complications, such as ureteric stricture, were documented during the follow-up period. Oral pain medication was used in $86 \%$ of the URS compared with $74 \%$ of ESWL cases $(\mathrm{p}=0.019)$, for a significantly longer duration $(2.4 \pm 1.5$ versus $1.9 \pm 1.5$ days, respectively, $\mathrm{p}=$ $0.029)$. Follow-up was significantly shorter for the URS group $(4.2 \pm 3.4$ versus $5.8 \pm 3.0$ weeks, $\mathrm{p}=$ 0.0001 ) (Table-3). Stone-free status at 1 month and 3 months were $93 \%$ and $97.5 \%$, respectively (Figure1). The mean satisfaction score was $4.03 \pm 2.08$ which is significantly different from the ESWL group $(\mathrm{p}=$ 0.043 ). Overall, 113 patients ( $94 \%$ ) were completely satisfied with the therapeutic modality chosen and will recommend it to the others except for the 7 patients who required re-treatment or open surgery and preferred to undergo ESWL for recurrence (Table-3).

\section{COMMENTS}

Ureteric stones have a high probability of spontaneous clearance. Spontaneous passage should be favored if possible $(11,13)$. According to a metaanalysis by the AUA Guidelines Panel, newly diagnosed stones with a diameter $<5 \mathrm{~mm}$ will pass in up to $98 \%$, depending on the degree of obstruction, urothelial edema and degree of impaction (11). With close controls and in absence of risk factors like impaired renal function, pain, urinary tract infection or fever, these stones can be followed safely until spontaneously cleared. However, most authors recommend not exceeding 4-6 weeks, especially for obstructive ureteric calculi $(14,15)$. These data show that the success rate is strongly influenced by the timing of therapeutic intervention (9). The sooner therapy is initiated, the more stones that might have passed spontaneously will be treated and, thus, false results in favor of the chosen procedure will be obtained. In particular small stones have a high spontaneous passage rate and so therapeutic intervention should be delayed to allow clearance (9). For this reason treatment was delayed in our study until 3 weeks after the diagnosis of a prevesical stone unless earlier therapeutic intervention was mandatory because of recurrent colic.

Peschel et al. (9) have reported on the differences that they have encountered in dealing with distal ureteral calculi with both ESWL and URS (rigid or semi-rigid). URS was significantly better in terms of shorter operative time, fluoroscopy time and time to achieve a stone free status. The authors recommend URS as first-line treatment for smaller stones $(<5$ $\mathrm{mm}$ ) that do not pass spontaneously.

In our series patient satisfaction was uniformly high in both groups but only significantly higher for URS (94\%) compared to shock wave lithotripsy $(80 \%)(\mathrm{p}=0.002)$. Also, patient willingness to undergo a repeated procedure of the same type favored URS. No true validated instrument exists for comparing patient symptoms and satisfaction with these different treatment options (16).

The efficacy of the treatment cannot be only judged by the stone free rate but various other parameters like postoperative symptoms, patient willingness to undergo a repeated procedure or to recommend it and the time of return to normal activity. The satisfaction criteria in this study were more extensive. In our series from the patient viewpoint achieving a stone-free state as soon as possible is the ultimate goal once the therapeutic approach has been chosen by most of the patients.

Patient satisfaction generally reflected treatment success. When assessing the efficacy of treatment an important consideration is the time it takes to achieve a stone free status._Peschel et al. (9) also concluded that in this respect there are considerable differences between ESWL and URS. Results of their patient assessment clearly demonstrated how important it is to achieve a stone free state early and even the patients who were free of symptoms said that the awareness of residual stone fragments and fear of colic were an ever present source of discomfort and restricted their ability to perform daily activities. Therefore, most patients in 
their study were satisfied with URS but would not be satisfied with ESWL. Pearle et al. (7) found no significant difference in postoperative symptoms between the 2 treatment groups despite the presence of a ureteral stent in virtually all patients who underwent URS but only $16 \%$ of the ESWL group. Their sample size may preclude statistical significance but there was a definite trend towards fewer symptoms in regard to bladder irritability with shock wave lithotripsy. The ESWL group used less pain medication for a shorter period compared with the URS group, and patient satisfaction slightly favored ESWL (7). They recommended ESWL with a HM3 lithotripter as first-line treatment for distal stones. In our series, oral pain medication was used by $74 \%$ of the ESWL group compared to $86 \%$ of the URS cases, $(p=0.019)$, and the duration of analgesic use was significantly longer in the URS group $(p=0.029)$. Despite this our patients favored URS because of the longer time to obtain a stone free status with the ESWL in addition to the other parameters in the questionnaire. In this respect our results are in agreement with those of Peschel et al. (9).

From a retrospective review of planned sameday discharge after ureteroscopy in 114 patients, Wills and Burns (17) concluded that ureteroscopy should be considered an outpatient procedure. They reported a $24 \%$ immediate admission rate, with about half the admissions for "social" reasons. The inclusion of social components within our routine assessment minimizes admission required for social reasons. Our patients have difficulty in transports as they live far away from the hospital.

Given the high success rates for both treatment modalities in our study, treatment success must also consider secondary outcome parameters, such as complications rates, patient satisfaction, procedural efficacy and cost. Complication rates are low for the treatment modalities. In neither the series of Pearle et al. (7) or Peschel et al. (9) did ureteral perforation or stricture occur in either treatment group. However, ureteral injury is an established, albeit rare, complication of URS that has never been reported to occur with in situ shock wave lithotripsy. Furthermore, complications associated with ESWL are generally mild and related to fragment passage. In our series, although not reaching statistical significance, an almost 3-fold increase in minor complications occurred with URS compared to ESWL. Consequently, ESWL is a marginally safer modality associated with few if any long-term sequelae.

However, the invasiveness of ureteroscopy cannot be neglected. Before the emergence of modern techniques for stone fragmentation and newer, betterdesigned ureteroscopes, complications like ureteric perforation and avulsion were not uncommon. A comprehensive review of acute endoscopic injuries reported in the literature from 1984 to 1992 identified 314 ureteric perforations that occurred in 5117 procedures $(6.1 \%)$ and complete ureteric avulsion in another 17 procedures, though infrequent, were documented $(0.3 \%)$ (18). Harmon et al. (19) observed a decrease in overall complications from $20 \%$ to $12 \%$ during a 10-year period which were attributed to smaller ureteroscopes and increased surgeon's experience. Schuster et al. (20) suggested a significant reduction in ureteric perforation with a less operative time and postoperative complications with the surgeon's experience. Proximal migration of stones occurred in 2 patients $(1.7 \%)$, which is less than what had been reported. $(21,22)$. With the emergence of flexible ureteroscopes, migrated stones could be retrieved with basket. However, these state-of-the-art ureteroscopes are fragile and experience in our center is still limited. We still use semi-rigid ureteroscopes for all ureteric calculi.

In our study, only 12 patients (10\%) of the URS group had a double-J ureteric stent inserted for high stone load while 29 patients $(24.2 \%)$ had ureteric catheters for 24 hours. This significantly reduces the occurrence of colic, hematuria and other complications of obstruction. In the majority of patients undergoing uncomplicated URS for removal of distal ureteral calculi postoperative discomfort is modest, lasts less than 2 days and is easily controlled with oral analgesics. Stricture formation has not been identified. Hence, we do not believe that routine placement of a ureteral stent following uncomplicated URS for a distal ureteral calculus is necessary. Routine placement of ureteral stent after ureteroscopic stone has been considered the standard of care in most centers but Denstedt et al. (23) performed a prospective trial of non-stented versus 
stented ureteroscopic lithotripsy, and concluded that patients without a stent have significantly fewer symptoms in the early post-operative period, while there were no differences in terms of complications and stone free status. In addition it is also important to notice that with ESWL, more follow-up visits to the clinic were required until a stone-free state was achieved and at each visit, the patient was exposed to radiation from plain radiography.

Some investigators concluded that prophylactic antibiotic during ESWL are unnecessary in patients whose urine before treatment was sterile (24), other studies showed that antibiotic prophylaxis with several agents can reduce the rate of bacteriuria significantly (25). Currently, many urologists routinely prescribe antibiotic prophylaxis to reduce the potential risk.

On the other hand, an important disadvantage of URS is that the procedure has to be performed under general or spinal anesthesia as compared to ESWL, which uses intravenous analgesia. This exposes the patient to the risks of anesthesia and makes it unfavorable to patient with significant medical problems but there are some reports on local anesthesia combined with intravenous sedation for URS $(26,27)$. From our series local anesthesia with intravenous sedation were sufficiently effective and safe in our patients with good tolerance. The need for anesthesia during ESWL depends largely on the energy source. While spark gap lithotripters (HM-3, MFL 5000) are highly effective, they are also more painful for the patient, whereas piezoelectric shock wave lithotripsy is associated to the least pain yet low efficacy. We could not find difficulty in stone localization under sedation with the Dornier Lithotripter S. We suggest that the choice of treatment modality for ureteric stones will depend on the patient since the expertise for both modalities are equally available. Patient's factors will include acceptance of invasive procedure, physical health and preference for earlier stone-free status.

The American Urological Association (AUA) Guidelines Panel (11) reported its recommendations for the treatment of ureteric stones. Although this report was clear in its recommendations of in situ shock wave lithotripsy for the treatment of small ureteral calculi, it was less clear for the large $(>1 \mathrm{~cm})$ upper ureteric stones. Although ESWL, URS, percutaneous stone extraction and open surgery were evaluated as different options; laparoscopic ureterolithotomy was not mentioned. Indeed, the previously mentioned treatment options have rendered open procedures a rarity in many hospitals (28). Open surgery was required for two of our patients with hard large stones. Sharma et al. (29) reported that open mini-access ureterolithotomy to be a safe and reliable minimally invasive procedure; its role is mainly confined to salvage for failed first-line stone treatments but in selected cases, where a poor outcome can be predicted from other methods, it is an excellent first-line treatment. Laparoscopy has the advantage of high probability of removing the entire stone in one procedure, exactly like open surgery.

Success rates for shock wave lithotripsy may differ according to the lithotriptor used. Average stone-free rate for cumulative shock wave lithotripsy series in the literature using an HM3 lithotriptor is slightly but consistently higher than that achieved with many second and third generation lithotripters and may influence the choice of treatment (30). It is important to stress that the results with shock wave lithotripsy are truly machine specific and cannot be translated to use with other lithotripters (31).

The Dornier Lithotripter $S$ that we use, proved in different series to be very effective in the treatment of renal and ureteral calculi (32). Though this is not randomized prospective study, matching the two groups in terms of age, sex and stone size and studying consecutive patients managed by the same group of urologists minimize patient selection bias.

In summary, ESWL offers minimalinvasiveness but a higher risk of treatment failure compared to URS which reaches immediate high stone free rates. ESWL is a marginally safer modality associated with few if any long-term sequelae. Treatment decisions have to be drawn individually taking into account patients preference, personal experience and local equipment. We believe that ureteroscopy is preferable to ESWL for treatment of distal ureteral calculi since it is significantly more efficient with higher patient satisfaction. 


\section{CONFLICT OF INTEREST}

None declared.

\section{REFERENCES}

1. Huffman JL, Bagley DH, Lyon ES: Treatment of distal ureteral calculi using rigid ureteroscope. Urology. 1982; 20: 574-7.

2. Chaussy C, Schmiedt E, Jocham D, Brendel W, Forssmann B, Walther V: First clinical experience with extracorporeally induced destruction of kidney stones by shock waves. J Urol. 1982; 127: 417-20.

3. Biyani CS, Cornford PA, Powell CS: Ureteroscopic holmium lasertripsy for ureteric stones. Initial experience. Scand J Urol Nephrol. 1998; 32: 92-3.

4. Watson GM, Wickham JE: Initial experience with a pulsed dye laser for ureteric calculi. Lancet. 1986; 1: 1357-8.

5. Marberger M, Hofbauer J, Turk C, Hobarth K, Albrecht W: Management of ureteric stones. Eur Urol. 1994; 25: 265-72.

6. el-Faqih SR, Husain I, Ekman PE, Sharma ND, Chakrabarty A, Talic R: Primary choice of intervention for distal ureteric stone: ureteroscopy or ESWL? Br J Urol. 1988; 62: 13-8.

7. Pearle MS, Nadler R, Bercowsky E, Chen C, Dunn M, Figenshau RS, et al.: Prospective randomized trial comparing shock wave lithotripsy and ureteroscopy for management of distal ureteral calculi. J Urol. 2001; 166: $1255-60$.

8. Kapoor DA, Leech JE, Yap WT, Rose JF, Kabler R, Mowad JJ: Cost and efficacy of extracorporeal shock wave lithotripsy versus ureteroscopy in the treatment of lower ureteral calculi. J Urol. 1992; 148: 1095-6.

9. Peschel R, Janetschek G, Bartsch G: Extracorporeal shock wave lithotripsy versus ureteroscopy for distal ureteral calculi: a prospective randomized study. J Urol. 1999; 162: 1909-12.

10. Segura JW: Ureteroscopy for lower ureteral stones. Urology. 1993; 42: 356-7.

11. Segura JW, Preminger GM, Assimos DG, Dretler SP, Kahn RI, Lingeman JE, et al.: Ureteral Stones Clinical Guidelines Panel summary report on the management of ureteral calculi. The American Urological Association. J Urol. 1997; 158: 1915-21.

12. Ather MH, Memon A: Therapeutic efficacy of Dornier MPL 9000 for prevesical calculi as judged by efficiency quotient. J Endourol. 2000; 14: 551-3.
13. Knoll T, Alken P, Michel MS: Progress in Management of Ureteric Stones. EAU Update Series. 2005; 3: 44-50.

14. Miller OF, Kane CJ: Time to stone passage for observed ureteral calculi: a guide for patient education. $\mathrm{J}$ Urol. 1999; 162: 688-90.

15. Tiselius HG, Ackermann D, Alken P, Buck C, Conort P, Gallucci M: Guidelines on urolithiasis. Eur Urol. 2001; 40: 362-71.

16. Shah DO, Matlaga BR, Assimos DG: Selecting Treatment for Distal Ureteral Calculi: Shock Wave Lithotripsy versus Ureteroscopy. Rev Urol. 2003; 5: 40-4.

17. Wills TE, Burns JR: Ureteroscopy: an outpatient procedure? J Urol. 1994; 151: 1185-7.

18. Stoller ML, Wolf J: Endoscopic ureteral injuries. In: McAnich JW (ed.), Traumatic and Reconstructive Urology. Philadelphia, WB Sanders. 1996; pp. 199205.

19. Harmon WJ, Sershon PD, Blute ML, Patterson DE, Segura JW: Ureteroscopy: current practice and longterm complications. J Urol. 1997; 157: 28-32.

20. Schuster TG, Hollenbeck BK, Faerber GJ, Wolf JS Jr: Complications of ureteroscopy: analysis of predictive factors. J Urol. 2001; 166: 538-40.

21. Fong YK, Ho SH, Peh OH, Ng FC, Lim PH, Quek PL, et al.: Extracorporeal shockwave lithotripsy and intracorporeal lithotripsy for proximal ureteric calculi-a comparative assessment of efficacy and safety. Ann Acad Med Singapore. 2004; 33: 80-3.

22. Kelly JD, Keane PF, Johnston SR, Kernohan RM: Laser lithotripsy for ureteric calculi: results in 250 patients. Ulster Med J. 1995; 64: 126-30.

23. Denstedt JD, Wollin TA, Sofer M, Nott L, Weir M, D'A Honey RJ: A prospective randomized controlled trial comparing nonstented versus stented ureteroscopic lithotripsy. J Urol. 2001; 165: 1419-22.

24. Ilker Y, Turkeri LN, Korten V, Tarcan T, Akdas A: Antimicrobial prophylaxis in management of urinary tract stones by extracorporeal shock-wave lithotripsy: is it necessary? Urology. 1995; 46: 165-7.

25. Claes H, Vandeursen R, Baert L: Amoxycillin/ clavulanate prophylaxis for extracorporeal shock wave lithotripsy-a comparative study. J Antimicrob Chemother. 1989; 24: 217-20.

26. Hosking DH, Smith WE, McColm SE: A comparison of extracorporeal shock wave lithotripsy and ureteroscopy under intravenous sedation for the management of distal ureteric calculi. Can J Urol. 2003; 10: $1780-4$. 
27. Miroglu C, Saporta L: Transurethral ureteroscopy: is local anesthesia with intravenous sedation sufficiently effective and safe? Eur Urol. 1997; 31: 36-9.

28. Anagnostou T, Tolley D: Management of ureteric stones. Eur Urol. 2004; 45: 714-21.

29. Sharma DM, Maharaj D, Naraynsingh V: Open mini-access ureterolithotomy: the treatment of choice for the refractory ureteric stone? BJU Int. 2003; 92: 614-6.

Correspondence address:

Dr. Ibrahim Fathi Ghalayini

Associate Professor of Urology

P.O. Box 940165, Amman, 11194, Jordan

Fax: + 009626 568-7422

E-mail: ibrahimg@just.edu.jo
30. Gettman MT, Segura JW: Management of ureteric stones: issues and controversies. BJU Int. 2005; 95: 85-93.

31. Hochreiter WW, Danuser H, Perrig M, Studer UE: Extracorporeal shock wave lithotripsy for distal ureteral calculi: what a powerful machine can achieve. J Urol. 2003; 169: 878-80.

32. Di Pietro C, Micali S, De Stefani S, Celia A, De Carne C, Bianchi G: Dornier Lithotripter S. The first 50 treatments in our department. Urol Int. 2004; 72: 48-51.

Accepted after revision:

July 24, 2006

\section{EDITORIAL COMMENT}

Ghalayini and colleagues have prospectively studied the efficacy and patients satisfaction in a comparative non -randomized study comparing ureteroscopy and shock wave lithotripsy for distal ureteric stone. An informed consent was taken and patients opted for one or the other treatment arm.

The authors have done this study quite amicably and should be congratulated for the honest description of the results. However, there are several factors that should be emphasized before incorporating their findings into every day clinical practice.

Efficacy of the treatment for distal ureteric stone is judged not only by stone free rate but other factors like need for re-treatment, ancillary procedure requirement and admission, all but the last are analyzed by efficient quotient (1-3). In this work $80 \%$ patient falling ureteroscopy required hospitalization, this is contrary to contemporary experience as admission following ureteroscopy for distal ureteric stone is only required in a small minority. Most often it is for social reason, lack of follow-up, heath care facility (home care, trained general practitioner etc) and less commonly for complications.

The other major difference between ureteroscopy and shock wave lithotripsy is the quantum of complications. The incidence of major complications like ureteric avulsion and ureteric perforations are fortunately rare but still a potential possibility. In the present work, the authors have found a very low incidence of complications in the 2 groups with no major complication. Need for anesthesia is another major difference between the 2 procedures. Although in women with distal ureteric calculi requiring treatment, ureteroscopy could be done under intravenous sedation, in men the better tolerance of SWL must be weighed against the higher success rate of ureteroscopy. If both treatment modalities are available, patients with small distal ureteric calculi, 
in whom ureteroscopy is likely to be successful, should be informed of and offered their choice of either treatment modality. Overall, the study adds nicely to rapidly growing body of evidence that ureteroscopy is a better option of treatment for stones moderately large to larger stones (3).

\section{REFERENCES}

1. Clayman R, McClennan B, Garvin T: Lithostar: An electromagnetic shock wave acoustic unit for extracorporeal lithotripsy. J. Endourol. 1989; 3:307.

2. Ather MH, Akhtar S: Appropriate cutoff for treatment of distal ureteral stones by single session in situ extracorporeal shock wave lithotripsy. Urology. 2005; 66: $1165-8$.

3. Ather MH, Memon A: Therapeutic efficacy of Dornier MPL 9000 for prevesical calculi as judged by efficiency quotient.J Endourol. 2000; 14: 551-3.

Dr. M. Hammad Ather

Section of Urology, Department of Surgery Aga Khan University

Karachi, Pakistan

E-mail: hammad.ather@aku.edu

treatment of distal ureteral calculi. However, shock wave lithotripsy with or without stent implantation is the treatment of choice in some centers. Studies suggest that either SWL or URS are useful options for the management of distal ureteral calculi. Ureteroscopic access is frequently useful for the management of ureteral calculi when shock wave lithotripsy is failed and for complex calculi because shock wave lithotripsy is not the ideal modality for the management of this kind of calculi. Several investigators do not advocate the use of shock wave lithotripsy for the treatment of distal and prevesicular stones due to difficult positioning of the patients for these procedures in which prone or modified sitting position is preferred in these situations. The advances in the fiber optic lens systems resulted in the manufacturing of smaller ureteroscopic instruments, which enabled widespread use of routine diagnostic and therapeutic procedures within the ureter and kidney. Open surgery is rarely preferred today but it remains as an option for a salvage procedure. Alternatively laparoscopic surgery is a minimally 
invasive option that can be used in circumstances where open surgery may have been indicated.

As this study showed URS and ESWL modalities share an overall high success rate with low morbidity and both modalities has also proven to be effective and safe therefore the selection of the optimal treatment for distal ureteral calculi remains one of the most controversial issues currently in endourology.

Although ureterecospic treatment is more invasive than ESWL the patient may achieve a stone free status with a single procedure. ESWL is less invasive but a drawback from the patients' perspective may be the long follow-up until a stone free state or the risk of a requirement for additional invasive procedures and retreatment need associated with ESWL. Conversely patient may favor ESWL because of fear of the anesthesia requirement associated with ureteroscopy and the possibility of a temporary ureteral stent implementation. ESWL can be done as an outpatient procedure with sedation.

ESWL is equivalent to URS for smaller stones (less than $1 \mathrm{~cm}$ ) but becomes significantly less efficient with larger stones. Generally ESWL was recommended for small and solitary stones, and URS for large or multiple stones. Not expectedly, smaller stones (less than $5 \mathrm{~mm}$ ) that had not passed spontaneously by 3 weeks can be more efficiently treated with URS, because they are the most difficult to localize and focus with ESWL.

A review of the literature revealed that the mean stone free rate for ESWL are 50-95\% and for ureteroscopy $96-100 \%$, retreatment rates are $27-50 \%$ for ESWL and $0.8-19 \%$ for ureteroscopy.
Recent studies suggest a tendency from noninvasive ESWL to ureteroscopy. As depicted in the current study patient satisfaction is also better in URS.

Choice of treatment modality depends on the current data regarding effectivity, complications and cost-effectiveness, physicians' expertise and available equipment. The patients preferences as anesthesia acceptance or deny and immediate cure expectations are also the factors that effecting the choice. The patient should be informed for the existing active treatment modalities and their relative benefits and risks.

\section{REFERENCES}

1. Ceylan K, Sunbul O, Sahin A, Gunes M: Ureteroscopic treatment of ureteral lithiasis with pneumatic lithotripsy: analysis of 287 procedures in a public hospital. Urol Res. 2005; 33: 422-5.

2. Kupeli B, Biri H, Isen K, Onaran M, Alkibay T, Karaoglan U, Bozkirli I: Treatment of ureteral stones: comparison of extracorporeal shock wave lithotripsy and endourologic alternatives. Eur Urol. 1998; 34: 4749.

3. Peschel R, Janetschek G, Bartsch G: Extracorporeal shock wave lithotripsy versus ureteroscopy for distal ureteral calculi: a prospective randomized study. J Urol. 1999; 162: 1909-12.

4. Shah DO, Matlaga BR, Assimos DG: Selecting treatment for distal ureteral calculi: shock wave lithotripsy versus ureteroscopy. Rev Urol. 2003; 5: 40-4.

\section{Dr. Kadir Ceylan \\ Department of Urology, School of Medicine Yuzuncu Yil University \\ Van, Turkey \\ E-mail:drceylan26@yahoo.com}

this issue, there have been only 2 prospective randomized trials to date, each with a contradictory answer. In a multi-institutional trial Pearle et al. (1) 
concluded that shock wave lithotripsy is preferable while Peschel et al. (2) instead determined that ureteroscopy should be first line treatment. Of note, these conclusions were not based on stone free rates alone, but instead included results from patient questionnaires addressing postoperative pain and satisfaction. Due to its non-randomized study design and inherent risk of selection bias, this work by Ghalayini and colleagues does not provide the definitive answer for the treatment of distal ureteral stones. However, it does provide an interesting insight into what patients find important regarding their procedure. Despite taking significantly less oral pain medication for a shorter period of time and having fewer complications, patients in the shock wave lithotripsy group had a lower level of satisfaction than patients undergoing ureteroscopy. It is important to note that the questionnaire used to obtain these results has not been validated, but it is clear that the global assessment of patient satisfaction was composed of more than just postoperative discomfort. The authors suggest that the decreased satisfaction in the shock wave lithotripsy group was due to the more prolonged time for stone passage relative to ureteroscopy. While no analysis was performed directly addressing this conclusion, shorter time to stone passage following ureteroscopy is a possible explanation as to why patients favored a procedure that was clearly more painful. Definitive proof supporting this conclusion will require further study, but when counseling patients on shock wave lithotripsy versus ureteroscopy for treatment of distal ureteral stones, the patient's feelings regarding stone passage time may help suggest one procedure over the other.

\section{REFERENCES}

1. Pearle MS, Nadler R, Bercowsky E, Chen C, Dunn M, Figenshau RS, et al.: Prospective randomized trial comparing shock wave lithotripsy and ureteroscopy for management of distal ureteral calculi. J Urol. 2001; 166: 1255-60.

2. Peschel R, Janetschek G, Bartsch G: Extracorporeal shock wave lithotripsy versus ureteroscopy for distal ureteral calculi: a prospective randomized study. J Urol. 1999; 162: 1909-12.

Dr. Kyle Anderson

Dept of Urology, Section of Endourology University of Minnesota

Edina, Minnesota, USA 